\title{
Acute Morbidity and Mortality Analysis of COVID-19 in Children Receiving Cancer Treatment
}

\author{
Najma Shaheen, Rabia Muhammed Wali, Haleema Saeed, Irfana Ishaq Sandhu, Maham Qaisar and Romeena \\ Qazi \\ Department of Pediatric Oncology, Shaukat Khanum Cancer Hospital, Lahore, Pakistan
}

\begin{abstract}
The aim of this study was to determine overall morbidity and mortality of COVID-19 infection in children on cancer treatment. It was an observational study, carried at Shaukat Khanum Cancer Hospital from $1^{\text {st }}$ April 2020 to $31^{\text {st }}$ July 2020 . A total of 165 children on active cancer treatment were tested for COVID-19 with PCR; out of these, 17 were detected positive. Twelve children were symptomatic having fever with or without cough, sore throat, body aches, rash or diarrhea. Two children had concurrent gram negative bacteremia. Ten children (58.8\%) required hospitalisation, $23.5 \%$ required oxygen and two had intensive care unit admission. One death was reported in this study. Chemotherapy was modified in five children, while elective surgery, chemotherapy and radiotherapy schedule were affected in eight children. Overall, the spread of Covid-19 was limited, the course of disease was mild, and anticancer treatment was provided and continued as per standard protocols.
\end{abstract}

Key Words: Covid-19, Cancer, Anticancer chemotherapy, Immunosuppression, Children.

How to cite this article: Shaheen N, Wali RM, Saeed H, Sandhu II, Qaisar M, Qazi R. Acute Morbidity and Mortality Analysis of COVID-19 in Children Receiving Cancer Treatment. J Coll Physicians Surg Pak 2021; 31(JCPSPCR):CR83-CR86.

\section{INTRODUCTION}

Novel coronavirus disease 2019 (COVID 19) has been declared as pandemic by World Health Organisation (WHO), initially revealed in Wuhan, China in November 2019. ${ }^{1}$ Paediatric series have reported low incidence of severe or critical illness in children with confirmed COVID-19. But high infectivity of virus, asymptomatic carriers, lack of herd immunity, and a wide range of presentations including mild disease to cytokine release storms and Kawasaki like multisystem inflammatory response are the major challenges. ${ }^{2}$

Children with cancer are more susceptible to infections due to malignancy itself and anticancer treatment, making immune system weak against respiratory bacteria and viruses and increasing the risk of severe events. ${ }^{3}$ There is limited data on the COVID19 infection phenotype in children on active anticancer treatment, its clinical outcome and case fatality.

The aim of this study was to determine overall morbidity and mortality of COVID-19 infection, clinical characteristics, and its impact on active treatment of children with cancer.

Correspondence to: Dr. Najma Shaheen, Department of Pediatric Oncology, Shaukat Khanum Cancer Hospital, Lahore, Pakistan

E-mail:dr_najmasaeed@yahoo.com

Received: October 18, 2020; Revised: February 18, 2021; Accepted: March 05, 2021

DOI: https://doi.org/10.29271/jcpsp.2021.JCPSPCR.CR83

\section{METHODOLOGY}

After approval from the Institutional Review Board, the Hospital-based electronic medical records (EMR) were retrospectively reviewed for the children, who were on active anticancer treatment at Hospital from $1^{\text {st }}$ April 2020 to $31^{\text {st }}$ July-2020, and were tested for COVID-19 by PCR.

All patients entering in the Hospital were triaged at dedicated desks for COVID-19 screening. All those with history of fever or respiratory signs and symptoms had nasopharyngeal swab for the detection of SARS-Cov-2. Newly diagnosed children with acute lymphoblastic leukemia (ALL), Burkitt's lymphoma (BL), and those who were undergoing intensive phase of chemotherapy had virus testing before commencement of treatment during this period. Children who were planned for elective surgery had COVID-19 testing at 24-48 hours before surgery. Children on active treatment of cancer with negative PCR for COVID-19 were excluded from this study.

Data were collected by reviewing EMR. Data fields included demographic variables, diagnosis, treatment category, clinical features with COVID-19 infection, duration of hospital stay, intensive care unit (ICU) admission, impact on treatment and outcome. This cohort of patients were followed till $31^{\text {st }}$ August, 2020. A case of COVID-19 was defined by the detection of SARSCov 2 RNA specific fragments by real time reverse transcriptase polymerase chain reaction (RT-PCR) from a nasopharyngeal swab.

Children were stratified according to age ( $<5,5-10$ and $>10$ years), underlying malignancy like leukemia/lymphoma and solid tumors. 
Table I: Patients' characteristics.

\begin{tabular}{|c|c|c|}
\hline Total number of children with COVID-19 PCR testing & 165 & \\
\hline COVID-19 PCR positive & $\begin{array}{c}\text { Number } \\
\text { (17) }\end{array}$ & $\begin{array}{c}\text { Percentage } \\
10.3 \%\end{array}$ \\
\hline $\begin{array}{l}\text { Age: } \\
<5 \text { years } \\
5-10 \text { years } \\
>10 \text { years }\end{array}$ & $\begin{array}{l}8 \\
3 \\
6\end{array}$ & $\begin{array}{l}47.1 \% \\
17.6 \% \\
35.3 \%\end{array}$ \\
\hline $\begin{array}{l}\text { Gender: } \\
\text { Male } \\
\text { Female }\end{array}$ & $\begin{array}{c}14 \\
3\end{array}$ & $\begin{array}{l}82.4 \% \\
17.6 \%\end{array}$ \\
\hline $\begin{array}{l}\text { Diagnosis } \\
\text { Leukemia/lymphoma } \\
\text { - Pre B Acute lymphoblastic leukemia (ALL) } 5 \\
\text { - Relapsed Pre B ALL } 1 \\
\text { - Burkitt lymphoma } 1 \\
\text { - Hodgkin lymphoma } 1 \\
\text { - Relapsed Hodgkin lymphoma } 1 \\
\text { Solid tumors } \\
\text { - Osteosarcoma } 3 \\
\text { - Wilms tumor } 1 \\
\text { - Rhabdomyosarcoma } 1 \\
\text { - Hepatoblastoma } 1 \\
\text { - Germ cell tumor } 1 \\
\text { - Relapsed Wilms tumor } 1\end{array}$ & 9 & $52.9 \%$ \\
\hline $\begin{array}{l}\text { Symptoms: } \\
\text { Asymptomatic } \\
\text { Fever/cough/diarrhoea/rash }\end{array}$ & $\begin{array}{c}5 \\
12 \\
\end{array}$ & $\begin{array}{l}29.4 \% \\
70.6 \% \\
\end{array}$ \\
\hline Acute neutrophil count (ANC) $<1$ & 7 & $41.2 \%$ \\
\hline Hospitalisation & 10 & $58.8 \%$ \\
\hline Oxygen requirement & 4 & $23.5 \%$ \\
\hline Intensive care unit admission & 2 & $11.8 \%$ \\
\hline Concurrent gram negative bacteremia & 2 & $11.8 \%$ \\
\hline No impact/modification of treatment & 4 & $23.5 \%$ \\
\hline Chemotherapy modification & 5 & $29.4 \%$ \\
\hline Delay in chemotherapy (range (7-14 days) & 3 & $17.6 \%$ \\
\hline Delay in elective surgery (range 11-20 days ) & 4 & $23.5 \%$ \\
\hline Delay in XRT and chemotherapy & 1 & $5.9 \%$ \\
\hline $\begin{array}{l}\text { Repeat COVID }-19 \text { testing at } 2 \text { weeks } \\
\text { Negative } 1 \\
\text { Positive } 3\end{array}$ & 4 & $23.5 \%$ \\
\hline
\end{tabular}

Statistical analysis was performed using SPSS software (version 20.0). Descriptive statistics were performed as frequencies and percentages for qualitative data.

\section{RESULTS}

During the study period, a total of 165 pediatric oncology patients were tested for COVID-19. Amongst these, 17 children were detected positive and all were on active anti-cancer therapy including chemotherapy and/or surgery and/or radiation. Patient characteristics are described in Table I.

In addition to the oncologic diagnosis, two children had additional comorbidity of renal disease (atrophic kidney and chronic renal disease). In terms of radiologic findings, CXR was done only in nine patients (who presented with cough) and it was reported as normal in five patients. One patient had mediastinal mass due to disease but lungs were unremarkable, and remaining three showed a variety of findings including diffuse infiltrates and patchy air space disease. Acute lymphocyte count was in the range of $0-2.99 \times 10^{3} / \mu \mathrm{l}$. D-dimer was high in four patients and coagulation profile was normal in six patients, in whom it was checked (not done in all patients).

The median duration of hospitalisation was 5 days with a range from 1 to 50 days. Symptomatic children were given oral azithromycin. One child of relapsed ALL had prolonged inpatient stay of 50 days. He was initially admitted for septic shock and later on developed COVID-19 infection with concurrent polymicrobial multidrug resistance bacteremia, and required ICU support for pressors.

There was one $(5.9 \%)$ death with COVID-19 related pneumonia. This child was newly diagnosed with pre $b$ ALL and presented with cough, fever, body aches and respiratory distress. X-ray chest had bilateral diffuse airway changes. His course was complicated by gram negative bacteremia, ICU admission, ventilatory support, and multiorgan failure, 
leading to death.

Five newly diagnosed and one child with relapsed Hodgkin lymphoma (HL) had COVID-19 infection at the outset. Child with relapsed $\mathrm{HL}$ presented with mediastinal widening and respiratory distress. He was given steroids as an emergency management, while awaiting confirmation of relapse and salvage chemotherapy.

\section{DISCUSSION}

Severe acute respiratory syndrome coronavirus 2 (SARS-CoV-2) has dominated worldwide and continues to escalate globally. Since the outbreak in January 2020, a number of studies have been published on the impact of virus had on children with cancer, modification of therapy and acute morbidity and mortality. ${ }^{4}$ The challenge to treat children with cancer remains due to the potential risk of delay in diagnosis and treatment, fear of seeking medical care by families due to infection and non-availability of medical services, especially in lower middle income countries (LMIC), like Pakistan. However most pediatric patients had mild symptoms without fever or pneumonia and recovered within 1-2 weeks after disease onset. ${ }^{5}$

In this study, the frequency of COVID-19 infection in patients on active cancer treatment was $10.3 \%$. Asymptomatic infection rate was $29.4 \%$, diagnosed on screening before elective surgical procedure or planned chemotherapy. Seventy point six percent $(70.6 \%)$ of patients had symptoms ranging from fever to no fever with or without cough, sore throat and body aches. A correspondence from Memorial Sloane Kettering Cancer Center (MSKCC), reported $11.2 \%$ positive COVID-19 results, with $32.5 \%$ symptomatic and $67.5 \%$ asymptomatic infections. Another study reported $2.5 \%$ infection rate of COVID-19. ${ }^{6,7}$

Hospitalisation rate was $58.8 \%$ in the presently reported patients with COVID-19, and this included all newly diagnosed children with acute leukemia and Burkitt's lymphoma that would be admitted for stabilisation and start of chemotherapy any way. Four $(23.5 \%)$ of these patients required oxygen and $2(11.8 \%)$ went to intensive care unit with one requiring ventilatory support. One newly diagnosed case of ALL died in ICU, who also had concurrent multi-drug resistant gram negative (Serratia marcescens) septicemia, that progressed to multi-organ failure (presented with prolonged history, delay in getting to a medical facility). The rest of the patients recovered and no worsening of symptoms was observed. Boulad et al., in his study of 20 children with cancer, showed that most patients with COVID-19 had mild symptoms and were managed at home and only one required non-critical care hospitalisation. This data also showed that overall morbidity of COVID-19 in pediatric cancer patients is low at 5\%; requiring hospitalisation for symptomatic patients. ${ }^{6}$ Data published so far seems to show that cancer does not appear to be a condition that worsens the SAR-CoV-2 infection outcomes in children.

In this study, four patients (23.5\%) had their chemotherapy regimen modification for initial two weeks and then continued on their respective standard treatment protocols. All these four patients had hematological malignancies ( $A L L$ and Non-Hodgkin lymphoma). Treatment modification done by giving prophase (low intensity chemotherapy) to these patients based on the international consensus done and published by leading childhood cancer organisations worldwide. ${ }^{8,9}$ A study published from Italy also modified treatment in their patients with hematological malignancies, but do not suggest changing the standard treatment protocols.

Outcomes in children with cancer are good. However in the COVID-19 pandemic, the major risks to cancer treatment are associated with strain on the medical services. ${ }^{10}$ Depending upon the conditions and available medical facilities among different countries, every possible effort be made to reduce the risk of COVID-19 infection in patients and caregivers through preventive strategies.

\section{CONCLUSION}

In this study, the spread of COVID-19 was limited, the course of disease was mild, recovery was uneventful, and we were able to provide and continue treatment as per standard protocols. Improved outcome, better epidemiological understanding of SARS-CoV-2 infection in children is warranted by close collaboration among national and international experts.

\section{CONFLICT OF INTEREST:}

The authors declared no conflict of interest.

\section{AUTHORS' CONTRIBUTION:}

NS: Conception, collection, interpretation of data and drafting.

RW: Writing of manuscript and critical revision.

HS: Interpretation of data and review of manuscript.

ISS: Data collection and manuscript review.

MQ: Data collection, design of work.

RQ: Data collection, conception.

\section{REFERENCES}

1. World Health Organisation. Coronavirus disease (COVID-19) Pandemic. Available at http://www.who.int/emergencies/ diseases/novel-coronavirus-2019. Accessed March 11, 2020.2 .

2. Waltuch T, Gill P, Zinns LE, Whitney R, Tokarski J, Tsung JW, et al. Features of COVID-19 post-infectious cytokine release syndrome in children presenting to the emergency department. Am J Emerg Med 2020; 38(10):2246.e3- 2246.e6. doi: 10.1016/j.ajem.2020.05.058.

3. Zhang A, Hu Q, Liu A, Wang S, Jin S, Wang Y, et al. Prevention of COVID-19 infection in a pediatric oncology ward in Wuhan. Pediatric BloodCancer 2020; 67(10): e28424. 
4. Wolfs, Tom FW, Andishe A, Adriana B, Ester MB, Simon B, et al. Childhood hematology SWG COVID-19 - Impact on childhood haematology patients. HemaSphere 2020; 4(5):p e465. doi: 10.1097/HS9.0000000000000465.

5. Shen K, Yang Y. Diagnosis and treatment of 2019 novel coronavirus infection in children: A pressing issue. World J Pediatr 2020; 16(3):219-221. doi: 10.1007/s12519-02000344-6.

6. Boulad F, Kamboj M, Bouvier N, Mauguen A, Kung AL. COVID-19 in children with cancer in New York city. Jama Oncol 2020; 6(9):1459-60. doi: 10.1001/jamaoncol.2020. 2028.

7. Girmenia C, Gentile G, Micozzi A, Petrucci L, Malaspina F, Di Prima A, et al. COVID-19 in patients with hematologic disorders undergoing therapy: Perspective of a large referral hematology center in rome. Acta Haematol 2020;
143(6):574-582. doi: 10.1159/000510769.

8. Sullivan M, Bouffet E, Rodriguez-Galindo C, Luna-Fineman S, Khan MS, Kearns $P$, et al. The COVID-19 pandemic: A rapid global response for children with cancer from SIOP, COG, SIOP-E, SIOP-PODC, IPSO, PROS, CCI, and St Jude Global. Pediatr Blood Cancer 2020; 67(7):e28409. doi: 10.1002/pbc.28409.

9. Vaitkeviciene G, Heyman M, Jonsson OG. Early morbidity and mortality in childhood acute lymphoblastic leukemia with very high white blood cell count. Leukemia 2013; 27(11):2259-62. doi: 10.1038/leu.2013.137.

10. Terenziani M, Massimino M, Biassoni V, Casanova M, Chiaravalli S, Ferrari A, et al. SARS-CoV-2 disease and children under treatment for cancer. Pediatr Blood Cancer 2020; 67(9):e28346. doi: 10. 1002/pbc.28346. doi: 10.1002/pbc. 28346 . 